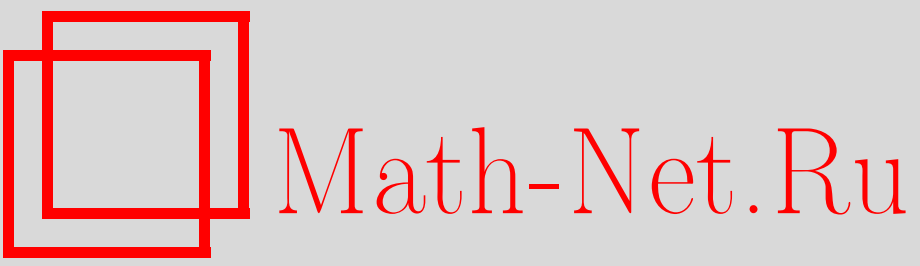

С. Озер, Е. Шухуби, Группы эквивалентности для уравнений баланса первого порядка и приложения к электромагнетизму, TM $\Phi$, 2003, том 137, номер 2, 271280

DOI: https://doi.org/10.4213/tmf271

Использование Общероссийского математического портала Math-Net.Ru подразумевает, что вы прочитали и согласны с пользовательским соглашением http://www . mathnet.ru/rus/agreement

Параметры загрузки:

IP : 52.205 .19 .152

26 апреля 2023 г., 02:24:17 


\section{ГРУППЫ ЭКВИВАЛЕНТНОСТИ ДЛЯ УРАВНЕНИЙ БАЛАНСА ПЕРВОГО ПОРЯДКА И ПРИЛОЖЕНИЯ К ЭЛЕКТРОМАГНЕТИЗМУ}

Исследуются группы преобразований эквивалентности для уравнений баланса первого порядка, включающих произвольное число зависимых и независимых переменных. Получены определяющие уравнения и найдены их явные решения. В основе используемого подхода лежит геометрический метод внешних дифференциальных форм Картана. Общие решения уравнений, задающих преобразования эквивалентности для систем первого порядка, применяются к уравнениям электродинамики Максвелла.

Ключевые слова: группы эквивалентности, изовекторный метод, уравнения баланса, уравнения Максвелла.

\section{1. ВВЕДЕНИЕ}

Рассмотрим общую систему дифференциальных уравнений в частных производных первого порядка, которая имеет вид уравнений баланса

$$
\frac{\partial \Sigma^{\alpha i}}{\partial u^{\beta}} u_{, i}^{\beta}+\frac{\partial \Sigma^{\alpha i}}{\partial x^{i}}+\Sigma^{\alpha}=0, \quad i=1,2, \ldots, n, \quad \alpha=1,2, \ldots, N
$$

где $\Sigma^{\alpha i}=\Sigma^{\alpha i}\left(x^{j}, u^{\beta}\right), \Sigma^{\alpha}=\Sigma^{\alpha}\left(x^{j}, u^{\beta}\right)$, через $x^{i}$ и $u^{\alpha}$ обозначены соответственно независимые и зависимые переменные, а индекс после запятой обозначает частную производную по соответствуюшей независимой переменной. Такие системы включают в себя широкий класс уравнений математической физики, поэтому их обшие решения легко могут быть приложены ко всем системам дифференциальных уравнений первого порядка, если определить $\Sigma^{\alpha i}$ и $\Sigma^{\alpha}$ подходящим образом.

В настоящей работе получены преобразования эквивалентности для системы вида (1). Мы получаем определяюшие уравнения и находим их точные решения. В последнем разделе в качестве приложения исследуются уравнения Максвелла.

\footnotetext{
* Department of Engineering Sciences, Faculty of Science and Letters, Istanbul Technical University, 80626 Maslak, Istanbul, Turkey. E-mail: ozers@itu.edu.tr

${ }^{\dagger}$ Department of Mathematics, Yeditepe University, 81120 Kayışdağ $\imath$, Istanbul, Turkey. E-mail: suhubi@yeditepe.edu.tr
} 
Группы эквивалентности - это группы непрерывных преобразований, которые отображают произвольное уравнение заданного семейства дифференциальных уравнений в другое уравнение того же семейства и преобразуют решение первого уравнения в решение второго:

$$
\begin{aligned}
\frac{\partial \Sigma^{\alpha i}(\mathbf{x}, \mathbf{u})}{\partial x^{i}}+\Sigma^{\alpha}(\mathbf{x}, \mathbf{u})=0 & \longrightarrow \frac{\partial \bar{\Sigma}^{\alpha i}(\overline{\mathbf{x}}, \overline{\mathbf{u}})}{\partial \bar{x}^{i}}+\bar{\Sigma}^{\alpha}(\overline{\mathbf{x}}, \overline{\mathbf{u}})=0, \\
\mathbf{u}=\mathbf{u}(\mathbf{x}) & \rightarrow \overline{\mathbf{u}}=\overline{\mathbf{u}}(\overline{\mathbf{x}}) .
\end{aligned}
$$

Геометрический метод, основанный на использовании внешних дифференциальных форм Картана [1], применяется для получения инфинитезимальных генераторов этих преобразований. Этот метод был впервые предложен Харрисоном и Эстабруком [2] в 1971 г. и был затем расширен Эделеном [3] и Шухуби [4].

\section{2. ОСНОВНЫЕ УРАВНЕНИЯ}

Пусть $M=\mathbb{R}^{n}$ обозначает пространство независимых переменных с декартовым координатным покрытием $\left\{x^{i}\right\}$ и $G=\mathbb{R}^{n} \times \mathbb{R}^{N}$ обозначает пространство графика с координатным покрытием $\left\{x^{i}, u^{\alpha}\right\}$. Для построения групп эквивалентности будем рассматривать функции $\Sigma^{\alpha i}$ и $\Sigma^{\alpha}$ как независимые переменные; чтобы учесть их функциональную зависимость, определим дополнительные переменные

$$
s_{j}^{\alpha i}=\frac{\partial \Sigma^{\alpha i}}{\partial x^{j}}, \quad \sigma_{\beta}^{\alpha i}=\frac{\partial \Sigma^{\alpha i}}{\partial u^{\beta}}, \quad t_{i}^{\alpha}=\frac{\partial \Sigma^{\alpha}}{\partial x^{i}}, \quad \tau_{\beta}^{\alpha}=\frac{\partial \Sigma^{\alpha}}{\partial u^{\beta}} .
$$

Рассмотрим расширенное многообразие $K$ с координатным покрытием

$$
\left\{x^{i}, u^{\alpha}, \Sigma^{\alpha i}, \Sigma^{\alpha}, s_{j}^{\alpha i}, \sigma_{\beta}^{\alpha i}, t_{i}^{\alpha}, \tau_{\beta}^{\alpha}\right\}
$$

Пусть $\Lambda(K)$ - внешняя алгебра на многообразии $K$. Определим далее контактные 1-формы и балансные $n$-фором следуюшим образом:

$$
\begin{aligned}
\Omega^{\alpha i} & =d \Sigma^{\alpha i}-s_{j}^{\alpha i} d x^{j}-\sigma_{\beta}^{\alpha i} d u^{\beta}, \\
\Omega^{\alpha} & =d \Sigma^{\alpha}-t_{i}^{\alpha} d x^{i}-\tau_{\beta}^{\alpha} d u^{\beta}, \\
\omega^{\alpha} & =d \Sigma^{\alpha i} \wedge \mu_{i}+\Sigma^{\alpha} \mu,
\end{aligned}
$$

где $\mu=d x^{1} \wedge d x^{2} \wedge \cdots \wedge d x^{n}$ - базисная форма в пространстве форм $\left.\Lambda^{n}(M), \mu_{i}=\partial / \partial x^{i}\right\rfloor \mu$ - базисные формы в пространстве форм $\Lambda^{n-1}(M)$, символ $\wedge$ обозначает внешнее произведение, а символ 」- внутреннее произведение.

Замкнутый идеал $I$ на расширенном многообразии $K$ порождается формами $\Omega^{\alpha i}, \Omega^{\alpha}$, $\omega^{\alpha}, d \Omega^{\alpha i}, d \Omega^{\alpha}$ и $d \omega^{\alpha}$. 


\section{3. НАХОЖДЕНИЕ ИЗОВЕКТОРНЫХ ПОЛЕЙ}

3.1. Характеристические уравнения. Общее векторное поле $V \in T(K)$ может быть записано в виде

$V=X^{i} \frac{\partial}{\partial x^{i}}+U^{\alpha} \frac{\partial}{\partial u^{\alpha}}+S^{\alpha i} \frac{\partial}{\partial \Sigma^{\alpha i}}+T^{\alpha} \frac{\partial}{\partial \Sigma^{\alpha}}+S_{j}^{\alpha i} \frac{\partial}{\partial s_{j}^{\alpha i}}+\mathcal{S}_{\beta}^{\alpha i} \frac{\partial}{\partial \sigma_{\beta}^{\alpha i}}+T_{i}^{\alpha} \frac{\partial}{\partial t_{i}^{\alpha}}+\mathcal{T}_{\beta}^{\alpha} \frac{\partial}{\partial \tau_{\beta}^{\alpha}}$.

Для того чтобы $V$ было изовекторным полем, необходимо и достаточно выполнение следуюшего условия:

$$
\alpha \in I \quad \Rightarrow \quad \mathcal{L}_{V} \alpha \in I
$$

Другими словами, производные Ли генераторов идеала $I$ должны оставаться в этом же идеале: определенные формулами (4) 1-формы и балансные $n$-формы должны удовлетворять условиям

$$
\begin{aligned}
\mathcal{L}_{V} \Omega^{\alpha i} & =L_{\beta j}^{\alpha i} \Omega^{\beta j}+L_{\beta}^{\alpha i} \Omega^{\beta} \\
\mathcal{L}_{V} \Omega^{\alpha} & =M_{\beta i}^{\alpha} \Omega^{\beta i}+M_{\beta}^{\alpha} \Omega^{\beta} \\
\mathcal{L}_{V} \omega^{\alpha} & =\nu_{\beta}^{\alpha} \omega^{\beta}+\Omega^{\beta i} \wedge C_{\beta i}^{\alpha}+d \Omega^{\beta i} \wedge D_{\beta i}^{\alpha}+\Omega^{\beta} \wedge C_{\beta}^{\alpha}+d \Omega^{\beta} \wedge D_{\beta}^{\alpha}
\end{aligned}
$$

с коэффициентами

$$
L_{\beta j}^{\alpha i}, L_{\beta}^{\alpha i}, M_{\beta i}^{\alpha}, M_{\beta}^{\alpha}, \nu_{\beta}^{\alpha} \in \Lambda^{0}(K), \quad C_{\beta i}^{\alpha}, C_{\beta}^{\alpha} \in \Lambda^{n-1}(K), \quad D_{\beta i}^{\alpha}, D_{\beta}^{\alpha} \in \Lambda^{n-2}(K) .
$$

Поскольку $\mathcal{L}_{V} d \alpha=d \mathcal{L}_{V} \alpha$, то нет необходимости вычислять $\mathcal{L}_{V} d \Omega^{\alpha i}, \mathcal{L}_{V} d \Omega^{\alpha}$ или $\mathcal{L}_{V} d \omega^{\alpha}$

Производная Ли контактной 1-фформы $\Omega^{\alpha i}$ может быть получена в виде

$$
\mathcal{L}_{V} \Omega^{\alpha i}=d F^{\alpha i}-S_{j}^{\alpha i} d x_{j}+X^{j} d s_{j}^{\alpha i}-\mathcal{S}_{\beta}^{\alpha i} d u_{\beta}+U^{\beta} d \sigma_{\beta}^{\alpha i}
$$

где мы обозначили

$$
F^{\alpha i}=S^{\alpha i}-X^{j} s_{j}^{\alpha i}-U^{\beta} \sigma_{\beta}^{\alpha i}
$$

и использовали известную формулу $\left.\left.\mathcal{L}_{V} \alpha=d(V\rfloor \alpha\right)+V\right\rfloor d \alpha$ дифференцирования Ли. Сравнивая обе части (7) и первое уравнение в (6), получаем

$$
\begin{gathered}
L_{\beta j}^{\alpha i}=\frac{\partial F^{\alpha i}}{\partial \Sigma^{\beta j}}, \quad L_{\beta}^{\alpha i}=\frac{\partial F^{\alpha i}}{\partial \Sigma^{\beta}}, \quad \frac{\partial F^{\alpha i}}{\partial t_{j}^{\beta}}=\frac{\partial F^{\alpha i}}{\partial \tau_{\gamma}^{\beta}}=0, \\
\mathcal{S}_{\beta}^{\alpha i}=\frac{\partial F^{\alpha i}}{\partial u^{\beta}}+\frac{\partial F^{\alpha i}}{\partial \Sigma^{\gamma j}} \sigma_{\beta}^{\gamma j}+\frac{\partial F^{\alpha i}}{\partial \Sigma^{\gamma}} \tau_{\beta}^{\gamma}, \quad S_{j}^{\alpha i}=\frac{\partial F^{\alpha i}}{\partial x^{j}}+\frac{\partial F^{\alpha i}}{\partial \Sigma^{\gamma k}} s_{j}^{\gamma k}+\frac{\partial F^{\alpha i}}{\partial \Sigma^{\beta}} t_{j}^{\beta}, \\
X^{j} \delta_{\beta}^{\alpha} \delta_{k}^{i}+\frac{\partial F^{\alpha i}}{\partial s_{j}^{\beta k}}=0, \quad U^{\beta} \delta_{\gamma}^{\alpha} \delta_{j}^{i}+\frac{\partial F^{\alpha i}}{\partial \sigma_{\beta}^{\gamma j}}=0 .
\end{gathered}
$$


Аналогичные вычисления для 1-форормы $\Omega^{\alpha}$ дают

$$
\begin{gathered}
M_{\beta i}^{\alpha}=\frac{\partial G^{\alpha}}{\partial \Sigma^{\beta i}}, \quad M_{\beta}^{\alpha}=\frac{\partial G^{\alpha}}{\partial \Sigma^{\beta}}, \quad \frac{\partial G^{\alpha}}{\partial s_{j}^{\beta i}}=\frac{\partial G^{\alpha}}{\partial \sigma_{\gamma}^{\beta i}}=0, \\
T_{i}^{\alpha}=\frac{\partial G^{\alpha}}{\partial x^{i}}+\frac{\partial G^{\alpha}}{\partial \Sigma^{\beta j}} s_{i}^{\beta j}+\frac{\partial G^{\alpha}}{\partial \Sigma^{\beta}} t_{i}^{\beta}, \quad \mathcal{T}_{\beta}^{\alpha}=\frac{\partial G^{\alpha}}{\partial u^{\beta}}+\frac{\partial G^{\alpha}}{\partial \Sigma^{\gamma i}} \sigma_{\beta}^{\gamma i}+\frac{\partial G^{\alpha}}{\partial \Sigma^{\gamma}} \tau_{\beta}^{\gamma}, \\
X^{i} \delta_{\beta}^{\alpha}+\frac{\partial G^{\alpha}}{\partial t_{i}^{\beta}}=0, \quad U^{\beta} \delta_{\gamma}^{\alpha}+\frac{\partial G^{\alpha}}{\partial \tau_{\beta}^{\gamma}}=0,
\end{gathered}
$$

где введено обозначение

$$
G^{\alpha}=T^{\alpha}-X^{i} t_{i}^{\alpha}-U^{\beta} \tau_{\beta}^{\alpha}
$$

Уравнения (11) и (14) приводят к суженным формам

$$
\begin{aligned}
X^{i} & =X^{i}\left(x^{j}, u^{\beta}, \Sigma^{\beta j}, \Sigma^{\beta}\right), & U^{\alpha} & =U^{\alpha}\left(x^{j}, u^{\beta}, \Sigma^{\beta j}, \Sigma^{\beta}\right), \\
S^{\alpha i} & =S^{\alpha i}\left(x^{j}, u^{\beta}, \Sigma^{\beta j}, \Sigma^{\beta}\right), & & T^{\alpha}=T^{\alpha}\left(x^{i}, u^{\beta}, \Sigma^{\beta i}, \Sigma^{\beta}\right) .
\end{aligned}
$$

Рассмотрим, наконец, балансную $n$-фформу $\omega^{\alpha}$. Производная Ли этой формы может быть легко получена в виде

$$
\mathcal{L}_{V} \omega^{\alpha}=T^{\alpha} \mu+\left(d S^{\alpha i}+\Sigma^{\alpha} d X^{i}\right) \wedge \mu_{i}-d X^{j} \wedge d \Sigma^{\alpha i} \wedge \mu_{j i}
$$

Чтобы вычислить выражение (18), нужно сначала, принимая во внимание соотношения (16), (17), вычислить дифференциалы $d X^{i}$ и $d S^{\alpha i}$, а затем заменить формы $d \Sigma^{\alpha i}$, $d \Sigma^{\alpha}$ на соответственно $\Omega^{\alpha i}, \Omega^{\alpha}$, используя тождества (4). В итоге, собирая аналогичные формы в обеих частях (18) и в третьем уравнении (6), мы сразу находим $D_{\beta i}^{\alpha}=0$ и $D_{\beta}^{\alpha}=0$, в то время как остальные члены принимают следующий вид:

$$
\begin{aligned}
{\left[A^{\alpha}-\right.} & \left.\nu_{\beta}^{\alpha}\left(\Sigma^{\beta}+s_{i}^{\beta i}\right)\right] \mu+\left(G_{\beta}^{\alpha i}+A_{\gamma}^{\alpha i} \tau_{\beta}^{\gamma}-\nu_{\gamma}^{\alpha} \sigma_{\beta}^{\gamma i}\right) d u^{\beta} \wedge \mu_{i} A_{\beta \gamma}^{\alpha i j} d u^{\beta} \wedge d u^{\gamma} \wedge \mu_{j i}+ \\
+ & \Omega^{\beta j} \wedge\left[\left(A_{\beta j}^{\alpha i}-\nu_{\beta}^{\alpha} \delta_{j}^{i}\right) \mu_{i}-C_{\beta j}^{\alpha}-\frac{\partial X^{k}}{\partial \Sigma^{\beta k}} \Omega^{\alpha i} \wedge \mu_{j i}+A_{\gamma \beta}^{\alpha i} d u^{\gamma} \wedge \mu_{j i}\right]+ \\
+ & \Omega^{\beta} \wedge\left[A_{\beta}^{\alpha i} \mu_{i}-C_{\beta}^{\alpha}-\frac{\partial X^{j}}{\partial \Sigma^{\beta}} \Omega^{\alpha i} \wedge \mu_{j i}-\frac{\partial X^{j}}{\partial \Sigma^{\beta}} \sigma_{\gamma}^{\alpha i} d u^{\gamma} \wedge \mu_{j i}\right]=0
\end{aligned}
$$

Функции $A^{\alpha}, A_{\beta}^{\alpha i}, G_{\beta}^{\alpha i}, A_{\beta j}^{\alpha i}, A_{\beta \gamma}^{\alpha i}$ и $A_{\beta \gamma}^{\alpha i j}$ в (19) определяются на основе компонент изо- 
векторного поля следующим образом:

$$
\begin{aligned}
A^{\alpha}= & T^{\alpha}+\frac{\partial S^{\alpha i}}{\partial x^{i}}+\Sigma^{\alpha} \frac{\partial X^{i}}{\partial x^{i}}+\left(\frac{\partial S^{\alpha i}}{\partial \Sigma^{\beta j}}+\Sigma^{\alpha} \frac{\partial X^{i}}{\partial \Sigma^{\beta j}}+\frac{\partial X^{k}}{\partial x^{k}} \delta_{\beta}^{\alpha} \delta_{j}^{i}-\frac{\partial X^{i}}{\partial x^{j}} \delta_{\beta}^{\alpha}\right) s_{i}^{\beta j}+ \\
& +\left(\frac{\partial S^{\alpha i}}{\partial \Sigma^{\beta}}+\Sigma^{\alpha} \frac{\partial X^{i}}{\partial \Sigma^{\beta}}\right) t_{i}^{\beta}-\frac{\partial X^{j}}{\partial \Sigma^{\beta k}}\left(s_{i}^{\beta k} s_{j}^{\alpha i}-s_{j}^{\beta k} s_{i}^{\alpha i}\right)-\frac{\partial X^{j}}{\partial \Sigma^{\beta}}\left(t_{i}^{\beta} s_{j}^{\alpha i}-t_{j}^{\beta} s_{i}^{\alpha i}\right), \\
A_{\beta}^{\alpha i}= & \frac{\partial S^{\alpha i}}{\partial \Sigma^{\beta}}+\Sigma^{\alpha} \frac{\partial X^{i}}{\partial \Sigma^{\beta}}-\frac{\partial X^{j}}{\partial \Sigma^{\beta}}\left(s_{j}^{\alpha i}-s_{k}^{\beta k} \delta_{j}^{i}\right), \\
G_{\beta}^{\alpha i}= & \frac{\partial S^{\alpha i}}{\partial u^{\beta}}+\Sigma^{\alpha} \frac{\partial X^{i}}{\partial u^{\beta}}+\left(\frac{\partial S^{\alpha i}}{\partial \Sigma^{\gamma j}}+\Sigma^{\alpha} \frac{\partial X^{i}}{\partial \Sigma^{\gamma j}}+\frac{\partial X^{k}}{\partial x^{k}} \delta_{\gamma}^{\alpha} \delta_{j}^{i}-\frac{\partial X^{i}}{\partial x^{j}} \delta_{\gamma}^{\alpha}\right) \sigma_{\beta}^{\gamma j}- \\
& -\frac{\partial X^{j}}{\partial u^{\beta}}\left(s_{j}^{\alpha i}-s_{k}^{\alpha k} \delta_{j}^{i}\right)-\frac{\partial X^{i}}{\partial \Sigma \gamma k}\left(\sigma_{\beta}^{\alpha j} s_{j}^{\gamma k}-\sigma_{\beta}^{\gamma k} s_{j}^{\alpha j}\right)+ \\
& +\frac{\partial X^{j}}{\partial \Sigma^{k}}\left(\sigma_{\beta}^{\alpha i} s_{j}^{\gamma k}-\sigma_{\beta}^{\gamma k} s_{j}^{\alpha i}\right)+\frac{\partial X^{j}}{\partial \Sigma^{\gamma}}\left(t_{j}^{\gamma} \sigma_{\beta}^{\alpha i}-t_{k}^{\gamma} \sigma_{\beta}^{\alpha k} \delta_{j}^{i}\right), \\
A_{\beta j}^{\alpha i}= & \frac{\partial S^{\alpha i}}{\partial \Sigma^{\beta j}}+\Sigma^{\alpha} \frac{\partial X^{i}}{\partial \Sigma^{\beta j}}-\frac{\partial X^{k}}{\partial x^{l}}\left(\delta_{k}^{i} \delta_{j}^{l}-\delta_{j}^{i} \delta_{k}^{l} \delta_{j}^{i}\right) \delta_{\beta}^{\alpha}-\frac{\partial X^{k}}{\partial \Sigma^{\beta j}}\left(s_{k}^{\alpha i}-s_{m}^{\alpha m} \delta_{k}^{j}\right)+ \\
& +\left(\frac{\partial X^{l}}{\partial \Sigma^{\gamma k}} s_{l}^{\gamma k} \delta_{j}^{i}-\frac{\partial X^{i}}{\partial \Sigma^{\gamma k}} s_{j}^{\gamma k}+\frac{\partial X^{k}}{\partial \Sigma^{\gamma}} t_{k}^{\gamma} \delta_{j}^{i}-\frac{\partial X^{i}}{\partial \Sigma^{\gamma}} t_{j}^{\gamma}\right) \delta_{\beta}^{\alpha}, \\
A_{\beta \gamma}^{\alpha i}= & -\frac{\partial X^{i}}{\partial \Sigma^{\gamma k}} \sigma_{\beta}^{\alpha k}-\left(\frac{\partial X^{j}}{\partial \Sigma^{\eta k}} \sigma_{\beta}^{\eta k}+\frac{\partial X^{i}}{\partial \Sigma^{\eta}} \tau_{\beta}^{\eta}+\frac{\partial X^{i}}{\partial u^{\beta}}\right) \delta_{\gamma}^{\alpha}, \\
A_{\beta \gamma}^{\alpha i j}= & -\frac{\partial X^{j}}{\partial u^{\beta}} \sigma_{\gamma}^{\alpha i}+\left(\frac{\partial X^{j}}{\partial \Sigma^{\eta k}} \sigma_{\gamma}^{\eta k}+\frac{\partial X^{j}}{\partial \Sigma^{\eta}} \tau_{\gamma}^{\eta}\right) \sigma_{\beta}^{\alpha i} .
\end{aligned}
$$

Поскольку коэффициенты при независимых формах в уравнении (19) должны обращаться в нуль, то определяющие уравнения могут быть получены в виде

$$
\begin{gathered}
A^{\alpha}=\nu_{\beta}^{\alpha}\left(\Sigma^{\beta}+s_{i}^{\beta i}\right), \\
G_{\beta}^{\alpha i}+A_{\gamma}^{\alpha i} \tau_{\beta}^{\gamma}-\nu_{\gamma}^{\alpha} \sigma_{\beta}^{\gamma i}=0, \\
A_{[\beta \gamma]}^{\alpha[i j]}=0,
\end{gathered}
$$

где $\nu_{\beta}^{\alpha}$ - произвольные функции, а квадратные скобки в индексах обозначают антисимметризацию по соответствующим индексам.

3.2. Нахождение изовекторных полей в явном виде. Рассмотрим сначала третье определяюшее уравнение в (21). Подставляя определения $A_{\beta \gamma}^{\alpha i j}$, заданные шестым выражением в (20), в третье определяющее уравнение (21), в явном виде получаем

$$
\begin{aligned}
-\frac{\partial X^{j}}{\partial u^{\beta}} & \sigma_{\gamma}^{\alpha i}+\left(\frac{\partial X^{j}}{\partial \Sigma^{\eta k}} \sigma_{\gamma}^{\eta k}+\frac{\partial X^{j}}{\partial \Sigma^{\eta}} \tau_{\gamma}^{\eta}\right) \sigma_{\beta}^{\alpha i}+\frac{\partial X^{i}}{\partial u^{\beta}} \sigma_{\gamma}^{\alpha j}-\left(\frac{\partial X^{i}}{\partial \Sigma^{\eta k}} \sigma_{\gamma}^{\eta k}+\frac{\partial X^{i}}{\partial \Sigma^{\eta}} \tau_{\gamma}^{\eta}\right) \sigma_{\beta}^{\alpha j}+ \\
+ & \frac{\partial X^{j}}{\partial u^{\gamma}} \sigma_{\beta}^{\alpha i}-\left(\frac{\partial X^{j}}{\partial \Sigma^{\eta k}} \sigma_{\beta}^{\eta k}+\frac{\partial X^{j}}{\partial \Sigma^{\eta}} \tau_{\beta}^{\eta}\right) \sigma_{\gamma}^{\alpha i}- \\
& -\frac{\partial X^{i}}{\partial u^{\gamma}} \sigma_{\beta}^{\alpha j}-\left(\frac{\partial X^{i}}{\partial \Sigma^{i k}} \sigma_{\beta}^{\eta k}+\frac{\partial X^{i}}{\partial \Sigma^{\eta}} \tau_{\beta}^{\eta}\right) \sigma_{\gamma}^{\alpha j}=0
\end{aligned}
$$


Поскольку компоненты изовекторного поля $X^{i}$ не зависят от переменных $\sigma_{\beta}^{\alpha i}$ и $\tau_{\beta}^{\alpha}$ (см. $(16))$, уравнение (22) является полиномиальным уравнением по переменным $\sigma_{\beta}^{\alpha i}$ и $\tau_{\beta}^{\alpha}$. Другими словами, коэффициенты при этих переменных должны обрашаться в нуль:

$$
\frac{\partial X^{i}}{\partial u^{\beta}}=0, \quad \frac{\partial X^{i}}{\partial \Sigma^{\beta j}}=0, \quad \frac{\partial X^{i}}{\partial \Sigma^{\beta}}=0
$$

т.е. компоненты изовектора имеют вид

$$
X^{i}=X^{i}\left(x^{j}\right)
$$

Принимая во внимание результат (23) и используя функции $A^{\alpha}, G_{\beta}^{\alpha i}$ и $A_{\gamma}^{\alpha i}$, заданные формулами (20), мы можем выписать первые два определяюших уравнения (21) в явном виде:

$$
\begin{aligned}
& T^{\alpha}+\frac{\partial S^{\alpha i}}{\partial x^{i}}+\Sigma^{\alpha} \frac{\partial X^{i}}{\partial x^{i}}+ \\
& \quad+\left(\frac{\partial S^{\alpha i}}{\partial \Sigma^{\beta j}}+\frac{\partial X^{k}}{\partial x^{k}} \delta_{\beta}^{\alpha} \delta_{j}^{i}-\frac{\partial X^{i}}{\partial x^{j}} \delta_{\beta}^{\alpha}\right) s_{i}^{\beta j}+\frac{\partial S^{\alpha i}}{\partial \Sigma^{\beta}} t_{i}^{\beta}-\nu_{\beta}^{\alpha}\left(\Sigma^{\beta}+s_{i}^{\beta i}\right)=0 \\
& \frac{\partial S^{\alpha i}}{\partial u^{\beta}}+\left(\frac{\partial S^{\alpha i}}{\partial \Sigma \gamma j}+\frac{\partial X^{k}}{\partial x^{k}} \delta_{\gamma}^{\alpha} \delta_{j}^{i}-\frac{\partial X^{i}}{\partial x^{j}} \delta_{\gamma}^{\alpha}-\nu_{\gamma}^{\alpha} \delta_{j}^{i}\right) \sigma_{\beta}^{\gamma j}+\frac{\partial S^{\alpha i}}{\partial \Sigma^{\beta}} \tau_{\beta}^{\gamma}=0
\end{aligned}
$$

Из уравнения (24) мы можем получить произвольные функции $\nu_{\beta}^{\alpha}$ в виде $\nu_{\beta}^{\alpha}=$ $\nu_{\beta}^{\alpha}\left(x^{i}, u^{\gamma}, \Sigma^{\gamma i}, \Sigma^{\gamma}, s_{j}^{\gamma i}, t_{i}^{\gamma}, \tau_{\beta}^{\gamma}\right)$. Ввиду этого уравнение (25) может рассматриваться как полиномиальное уравнение по переменной $\sigma_{\beta}^{\alpha i}$ и может быть легко представлено в более простом виде:

$$
\frac{\partial S^{\alpha i}}{\partial u^{\beta}}=0, \quad \frac{\partial S^{\alpha i}}{\partial \Sigma^{\beta}}=0, \quad \frac{\partial S^{\alpha i}}{\partial \Sigma^{\gamma j}}+\left(\frac{\partial X^{k}}{\partial x^{k}} \delta_{j}^{i}-\frac{\partial X^{i}}{\partial x^{j}}\right) \delta_{\gamma}^{\alpha}=\nu_{\gamma}^{\alpha} \delta_{j}^{i} .
$$

Из третьего уравнения в (26) ясно, что

$$
\nu_{\beta}^{\alpha}=\frac{1}{n} \frac{\partial S^{\alpha i}}{\partial \Sigma^{\beta j}}+\frac{n-1}{n} \frac{\partial X^{i}}{\partial x^{i}} \delta_{\beta}^{\alpha} .
$$

Подставляя это выражение в третье уравнение (26) и определяя функцию

$$
f_{\beta}^{\alpha}=\nu_{\beta}^{\alpha}-\frac{\partial X^{i}}{\partial x^{i}} \delta_{\beta}^{\alpha}
$$

имеем

$$
\frac{\partial S^{\alpha i}}{\partial \Sigma^{\beta j}}=f_{\beta}^{\alpha} \delta_{j}^{i}+\frac{\partial X^{i}}{\partial x^{j}} \delta_{\beta}^{\alpha} .
$$

Дифференцируя по переменной $\Sigma^{\gamma k}$, в силу симметрии левой части легко можем записать

$$
\frac{\partial f_{\beta}^{\alpha}}{\partial \Sigma \gamma k} \delta_{j}^{i}=\frac{\partial f_{\gamma}^{\alpha}}{\partial \Sigma^{\beta j}} \delta_{k}^{i}
$$


Сворачивая по индексам $i$ и $j$, легко получаем $f_{\beta}^{\alpha}=f_{\beta}^{\alpha}\left(x_{i}\right)$. Следовательно, мы имеем $S^{\alpha i}$ в виде

$$
S^{\alpha i}=f_{\beta}^{\alpha}(\mathbf{x}) \Sigma^{\beta i}+\frac{\partial X^{i}}{\partial x^{j}} \Sigma^{\alpha j}+g^{\alpha i}(\mathbf{x})
$$

Последний шаг состоит в получении компонент изовекторного поля из уравнения (24):

$$
T^{\alpha}=f_{\beta}^{\alpha}(\mathbf{x}) \Sigma^{\beta}-\frac{\partial f_{\beta}^{\alpha}(\mathbf{x})}{\partial x^{i}} \Sigma^{\beta i}-\frac{\partial^{2} X^{i}}{\partial x^{i} \partial x^{j}} \Sigma^{\alpha j}-\frac{\partial g^{\alpha i}(\mathbf{x})}{\partial x^{i}},
$$

где $f_{\beta}^{\alpha}(\mathbf{x})$ и $g^{\alpha i}(\mathbf{x})$ являются произвольными функциями своих аргументов. Таким образом, все значимые компоненты изовекторов получены:

$$
\begin{aligned}
X^{i} & =X^{i}\left(x^{i}\right), \\
U^{\alpha} & =U^{\alpha}\left(x^{i}, u^{\alpha}, \Sigma^{\alpha i}, \Sigma^{\alpha}\right), \\
S^{\alpha i} & =f_{\beta}^{\alpha}\left(x^{j}\right) \Sigma^{\beta i}+\frac{\partial X^{i}}{\partial x^{j}} \Sigma^{\alpha j}+g^{\alpha i}\left(x^{j}\right), \\
T^{\alpha} & =f_{\beta}^{\alpha}\left(x^{i}\right) \Sigma^{\beta}-\frac{\partial f_{\beta}^{\alpha}\left(x^{j}\right)}{\partial x^{i}} \Sigma^{\beta i}-\frac{\partial^{2} X^{i}}{\partial x^{i} \partial x^{j}} \Sigma^{\alpha j}-\frac{\partial g^{\alpha i}\left(x^{i}\right)}{\partial x^{j}} .
\end{aligned}
$$

Группа эквивалентности, порождаемая некоторым конкретным изовекторным полем, может быть теперь найдена посредством интегрирования следуюшей системы обыкновенных дифференциальных уравнений относительно группового параметра $\varepsilon$ :

$$
\begin{aligned}
\frac{d \bar{x}^{i}}{d \varepsilon} & =X^{i}\left(\bar{x}^{j}\right), & \frac{d \bar{u}^{\alpha}}{d \varepsilon} & =U^{\alpha}\left(\bar{x}^{j}, \bar{u}^{\beta}, \bar{\Sigma}^{\beta j}, \bar{\Sigma}^{\beta}\right), \\
\frac{d \bar{\Sigma}^{\alpha i}}{d \varepsilon} & =S^{\alpha i}\left(\bar{x}^{j}, \bar{u}^{\beta}, \bar{\Sigma}^{\beta j}, \bar{\Sigma}^{\beta}\right), & \frac{d \bar{\Sigma}^{\alpha}}{d \varepsilon} & =T^{\alpha}\left(\bar{x}^{j}, \bar{u}^{\beta}, \bar{\Sigma}^{\beta j}, \bar{\Sigma}^{\beta}\right)
\end{aligned}
$$

с начальными условиями

$$
\bar{x}^{i}(0)=x^{i}, \quad \bar{u}^{\alpha}(0)=u^{\alpha}, \quad \bar{\Sigma}^{\alpha i}(0)=\Sigma^{\alpha i}, \quad \bar{\Sigma}^{\alpha}(0)=\Sigma^{\alpha} .
$$

Дополнительные компоненты изовектора легко могут быть вычислены из уравнений (10) и (13) с использованием определений (8) и (15). Если функции $\Sigma^{\alpha i}$ и/или $\Sigma^{\alpha}$ не зависят от некоторых переменных $x^{i}$ или $u^{\alpha}$, то компоненты изовектора, связанные с этими переменными, также должны обрашаться в нуль. Такие ограничения, наложенные на компоненты изовектора, сужают произвол в функциях, входящих в выражения для определенных выше компонент. 


\section{4. ПРИМЕНЕНИЕ К УРАВНЕНИЯМ МАКСВЕЛЛА}

Рассмотрим уравнения Максвелла в виде

$$
\begin{aligned}
e_{i j k} E_{k, j}+\dot{B}_{i}=0, & B_{i, i}=0, \\
e_{i j k} H_{k, j}-\dot{D}_{i}-J_{i}=0, & D_{i, i}=q_{f},
\end{aligned}
$$

где индексы $i, j$ и $k$ нумеруют пространственные координаты, $e_{i j k}-$ абсолютно антисимметричньй тензор, индекс после запятой обозначает частную производную по соответствующей пространственной координате и точка означает дифференцирование по времени. Здесь $E_{i}, H_{i}, D_{i}$ и $B_{i}$ являются соответственно компонентами векторов напряженности электрического поля, напряженности магнитного поля, электрической индукции и магнитной индукции, $J_{i}$ - компоненты вектора плотности электрического тока и $q_{f}$ - плотность свободного заряда. Система (28) содержит 15 неизвестных и только восемь уравнений. Следовательно, эта система является недоопределенной и должна быть дополнена уравнениями состояния, которые описьвают физические свойства материальной среды. В рамках настояшей работы мы рассматриваем несколько упрошенные строго нелинейные однородные уравнения состояния

$$
D_{i}=D_{i}\left(E_{j}\right), \quad B_{i}=B_{i}\left(H_{j}\right), \quad J_{i}=J_{i}\left(E_{j}\right), \quad q_{f}=q_{f}\left(x_{i}, t\right) .
$$

Для того чтобы представить уравнения Максвелла (28) в виде уравнений баланса (1), подставим в качестве независимых переменных $x^{i}$ переменные $\left\{x_{i}, t\right\}, i=1,2,3$, в качестве зависимых переменных $u^{\alpha}$ - переменные $\left\{u_{\alpha}=E_{\alpha}, u_{a}=H_{(a-3)}\right\}, \alpha=1,2,3$, $a=4,5,6$, и определим

$$
\begin{aligned}
& \Sigma_{\alpha j}=e_{\alpha j k} E_{k}, \quad \Sigma_{\alpha 4}=\delta_{\alpha j} B_{j}, \quad \Sigma_{\alpha}=0, \\
& \Sigma_{a j}=e_{(a-3) j k} H_{k}, \quad \Sigma_{a 4}=-\delta_{(a-3) j} D_{j}, \quad \Sigma_{a}=-\delta_{(a-3) j} J_{j}, \\
& \Sigma_{7 j}=B_{j}, \quad \Sigma_{74}=0, \quad \Sigma_{7}=0, \\
& \Sigma_{8 j}=D_{j}, \quad \Sigma_{84}=0, \quad \Sigma_{8}=-q_{f} .
\end{aligned}
$$

Согласно уравнениям состояния (29) некоторые из дополнительных переменных, задаваемых формулами (2), обрашаются в нуль:

$$
\begin{gathered}
s_{\alpha j i}=s_{\alpha j 4}=s_{\alpha 4 i}=s_{\alpha 44}=s_{a j i}=s_{a j 4}=s_{a 4 i}=s_{a 44}=s_{7 j i}=s_{7 j 4}=s_{8 j i}=s_{8 j 4}=0, \\
\sigma_{\alpha j n}=\sigma_{\alpha 4 m}=\sigma_{a j m}=\sigma_{a 4 n}=\sigma_{7 j m}=\sigma_{8 j n}=0, \\
t_{\alpha i}=t_{\alpha 4}=t_{7 i}=t_{74}=t_{a i}=t_{a 4}=0, \\
\tau_{\alpha m}=\tau_{\alpha n}=\tau_{a n}=\tau_{7 m}=\tau_{7 n}=\tau_{8 m}=\tau_{8 n}=0,
\end{gathered}
$$

где

$$
\begin{aligned}
\sigma_{\alpha j m} & =e_{\alpha j m}, & \sigma_{a j n} & =e_{(a-3) j n} \\
\sigma_{\alpha 4 n} & =\delta_{\alpha i} \sigma_{7 i m}, & \sigma_{a 4 m} & =-\delta_{(a-3) i} \sigma_{8 i m}
\end{aligned}
$$


Здесь нижний индекс $m$ обозначает частные производные по компонентам вектора напряженности электрического поля, а нижний индекс $n$ - частные производные по компонентам вектора напряженности магнитного поля. Компоненты изовекторов, соответствуюших обрашающимся в нуль переменным, также должны обрашаться в нуль:

$$
\begin{aligned}
S_{\alpha j i} & =S_{\alpha j 4}=S_{\alpha 4 i}=S_{\alpha 44}=S_{a j i}=S_{a j 4}=S_{a 4 i}=S_{a 44}= \\
& =S_{7 j i}=S_{7 j 4}=S_{8 j i}=S_{8 j 4}=0 \\
\mathcal{S}_{\alpha j m} & =\mathcal{S}_{\alpha j n}=\mathcal{S}_{\alpha 4 m}=\mathcal{S}_{a j m}=\mathcal{S}_{a j n}=\mathcal{S}_{a 4 n}=\mathcal{S}_{7 j m}=\mathcal{S}_{8 j n}=0 \\
T_{\alpha i} & =T_{\alpha 4}=T_{7 i}=T_{74}=T_{a i}=T_{a 4}=0 \\
\mathcal{T}_{\alpha m} & =\mathcal{T}_{\alpha n}=\mathcal{T}_{a n}=\mathcal{T}_{7 m}=\mathcal{T}_{7 n}=\mathcal{T}_{8 m}=\mathcal{T}_{8 n}=0 .
\end{aligned}
$$

Определения (30) требуют рассмотрения следующих соотношений между компонентами изовекторов:

$$
\begin{aligned}
T_{\alpha}=T_{7}=S_{74} & =S_{84}=0, \\
\mathcal{E}_{k}=\frac{1}{2} e_{\alpha j k} S_{\alpha j}, \quad \mathcal{H}_{k} & =\frac{1}{2} e_{(a-3) j k} S_{a j}, \\
\mathcal{B}_{i}=S_{7 i}=\delta_{\alpha i} S_{\alpha 4}, \quad \mathcal{D}_{i} & =S_{8 i}=-\delta_{(a-3) i} S_{a 4}, \\
T_{a}=-\delta_{(a-3) i} \mathcal{J}_{i}, \quad T_{8} & =-Q
\end{aligned}
$$

где $\mathcal{E}_{i}, \mathcal{H}_{i}, \mathcal{B}_{i}, \mathcal{D}_{i}, \mathcal{J}_{i}$ и $Q$ обозначают компоненты изовекторного поля, отвечающие $E_{i}$, $H_{i}, B_{i}, D_{i}, J_{i}$ и $q_{f}$, соответственно. Все компоненты изовекторного поля могут быть легко получены при помоши формул (27) с учетом соотношений (32). Произвольные функции, при обозначении которых в (27) использованы буквы $f$ и $g$, определяются с использованием ограничений (31) и первого уравнения в (32).

В качестве простого примера мы рассмотрим эффект, к которому приводит обращение в нуль компоненты $\mathcal{S}_{\alpha 4 m}$. Из первого уравнения в $(10)$ следует, что

$$
\mathcal{S}_{\alpha 4 m}=\frac{\partial F_{\alpha 4}}{\partial E_{m}}+\frac{\partial F_{\alpha 4}}{\partial \Sigma_{\beta j}} e_{\beta j m}+\frac{\partial F_{\alpha 4}}{\partial \Sigma_{a 4}} \sigma_{a 4 m}+\frac{\partial F_{\alpha 4}}{\partial \Sigma_{8 i}} \sigma_{8 i m}+\frac{\partial F_{\alpha 4}}{\partial \Sigma_{a}} \tau_{a m}=0
$$

Здесь, как ясно из $(8), F_{\alpha 4}=S_{\alpha 4}-\mathcal{H}_{n} \sigma_{\alpha 4 n}$. Если подставить выражения для $\mathcal{H}_{n}$ из $(32)$ и $S_{\alpha 4}$ из $(27)$ и ввести второе ограничение из (31), то обрашаюшиеся в нуль компоненты (33) порождают следуюшие ограничения:

$$
\begin{array}{ll}
T_{, j}=0, & f_{\alpha a}=0, \\
e_{(a-3) j n}\left(f_{a 8} \delta_{i j}-\dot{\phi}_{j} \delta_{(a-3) i}\right)=0, & f_{a \alpha}=0,
\end{array}
$$

где $\phi_{i}$ - компоненты изовектора по переменной $x_{i}$, а $T$ - компонента изовектора по времени. 
Аналогично, использовав все ограничения (31) и первое уравнение в (32), находим в конечном счете значимые компоненты изовекторного поля в виде

$$
\begin{aligned}
T & =\alpha_{1} t+\alpha_{2}, & \phi_{i} & =a_{i j} x_{j}+a_{i}, \\
\mathcal{B}_{i} & =b_{i j} B_{j}+b_{i}, & \mathcal{D}_{i} & =d_{i j} D_{j}+d_{i}, \\
\mathcal{E}_{i} & =\epsilon_{i j} E_{j}+\epsilon_{i}, & \mathcal{H}_{i} & =h_{i j} H_{j}+h_{i}, \\
\mathcal{J}_{i} & =n_{i j} J_{j}, & Q & =-\alpha q_{f},
\end{aligned}
$$

где все тензорные и векторные коэффициенты постоянны и должны удовлетворять соотношениям

$$
\begin{aligned}
e_{i j k} \epsilon_{k m}+e_{i j m} \alpha_{1}-e_{i k m} a_{j k}-e_{k j m} b_{i k} & =0, \\
e_{k j m} d_{i k}-e_{i j k} h_{k m}-e_{i j m} \alpha_{1}+e_{i k m} a_{j k} & =0, \\
\alpha \delta_{i j}=d_{i j}-a_{i j}, \quad e_{j i k}\left(n_{i j}+a_{j i}\right) & =0 .
\end{aligned}
$$

\section{Список литературы}

[1] Э. Картан. Внешние дифференциальные формы и их геометрические приложения. М.: Изд-во МГУ, 1962.

[2] B. K. Harrison, F. B. Estabrook. J. Math. Phys. 1971. V. 12. P. 653.

[3] D. G. B. Edelen. Applied Exterior Calculus. New York: Wiley, 1985; Isovector Methods for Equations of Balance. Alphen aan den Rijn: Noordhoff, 1980.

[4] E. S. Şuhubi. Internat. J. Engrg. Sci. 1991. V. 29. P. 133; 1999. V. 37. P. 1901; 2000. V. 38. P. 715 . 